\title{
Tracking Occluded Objects Using Kalman Filter and Color Information
}

\author{
Malik M. Khan, Tayyab W. Awan, Intaek Kim, and Youngsung Soh
}

\begin{abstract}
Robust visual tracking is imperative to track multiple occluded objects. Kalman filter and color information tracking algorithms are implemented independently in most of the current research. The proposed method combines extended Kalman filter with past and color information for tracking multiple objects under high occlusion. The proposed method is robust to background modeling technique. Object detection is done using spatio-temporal Gaussian mixture model (STGMM). Tracking consists of two steps: partially occluded object tracking and highly occluded object tracking. Tracking partially occluded objects, extended Kalman filter is exploited with past information of object, whereas for highly occluded object tracking, color information and size attributes are used. The system was tested in real world application and successful results were obtained.
\end{abstract}

Index Terms-EKF with color, tracking occluded objects, STGMM, robust tracking using color information.

\section{INTRODUCTION}

Object tracking is the process of following the position and status of an object. Visual tracking systems have served well in the field of video surveillance, militarily guidance, robot navigation, artificial intelligence and medical applications during the last two decades. The fundamental requirement for any vision based tracking system is its robustness to the variability in the visual data presentation by dynamic, uncontrolled environment. Most of the cases there are more than one object to track. Tracking multiple objects is still a challenging task when they are occluded [1]. The overall tracking performance depends on the precise extraction and pinpointing the position of the moving objects from the surveillance video.

Tracking initializes with extracting objects. Commonly-implemented background modeling techniques [2] could only perform well until there is a uniform motion i.e. camera jittering or a non-uniform motion such as flag fluttering, water rippling and swaying tree branches. Therefore, we need a robust technique which is dynamic and invulnerable to uniform or non-uniform motion in the background. The technique should use temporal as well as spatio-temporal relations. Such technique spatio-temporal Gaussian mixture model (STGMM) is presented Soh et al. [3] which is used in our work. After extraction, a nonlinear filter can help to keep the precise track of the objects.

Manuscript received December 10, 2013; revised February 9, 2014. This work (Grants No. C0005448) was supported by Business for Cooperative R\&D between Industry, Academy, and Research Institute funded by Korea Small and Medium Business Administration in 2012.

Malik M. Khan, T. W. Awan, I. Kim and Y. Soh are with Myongji University, Yongin, Korea (e-mail: kit@mju.ac.kr).
Therefore, extended Kalman filter (EKF) [4] is used to predict and update the state of the object.

In this work, a novel approach for tracking occluded objects is presented, which tracks multiple objects efficiently even if the background modeling is compromised at some instant. This paper is divided into four parts i.e. background modeling, extended Kalman filtering, dominant color information extraction and feature extraction. First of all, STGMM is applied to extract foreground. The proposed STGMM excludes the shadow and noise from the scene. Secondly, to predict the state of nonlinear objects EKF is exploited. The overall performance of the tracking system can be reinforced using EKF if the object is not extracted in one or more frames. Dominant color information extraction of each object is done in third step and utilized under confused situation i.e. occlusion of interested object by other objects. At last, the attributes of objects i.e. its track, color, time of appearance and leaving the scene and object kind are extracted and stored in separate data files for each object, which can later facilitate inquiring a particular object with certain color and object kind from the surveillance video.

\section{RELATED WORKS}

Numerous techniques have been proposed for multiple object tracking. However, in this section only few well-known techniques have been described in two different aspects: 1) Kalman filtering for objects tracking; and 2) Color matching for confused situations like occlusion.

\section{A. Tracking Using Kalman Filters}

Kalman filter recursively estimates the state of the target object. Kalman filtering is vastly used in different domains like object tracking, economics and navigation systems. But here we would only review it for object tracking.

A new method is presented by Liu et al. [5] which combines properties of EKF and unscented Kalman filter (UKF) for non-linear object tracking. Here, EKF is kept conventional but the deterministic sample is taken by unscented transformation. Then posterior mean of nonlinearity is noted by propagating sample, but the posterior covariance of nonlinearity is kept linear.

Berclaz et al. [6] propose an algorithm for frame-by-frame detection and linking the trajectories of an unknown number of targets for multi-object tracking using K-shortest path optimization.

Zhai et al. [7] propose an approach to track an object by a dynamic model from a finite set of models. As the single-model assumption could cause tracker unstable if the target has complex trajectory or the camera has abrupt ego-motions. 


\section{B. Color Information Matching}

Among well describable featured-matching properties i.e., shape, color and temporal, color serves well to distinguish between objects under confused situations.

A tracking scheme proposed by Zulfiqar et al. [8] employs particle filter and multi-mode anisotropic mean shift algorithms. They track the object using only 15 particles which increase the computational speed. The proposed method eliminates the track drift and loss of track during occlusion.

A novel approach for robust object tracking has been brought by Li et al. [9]. They track more than three occluded objects using dominant color histogram. Moreover, the selected colors are based on the given distance measure which is also robust to illumination change.

A multiple object tracking algorithm is presented by Xing et al. [1] which contributes in both observation modeling and tracking strategy level. For the observation modeling, the progressive observation model is presented and dual-mode two-way Bayesian is used for tracking strategy. The weighting factors in the proposed algorithm are color, size and motion cue. They not only locate dominant playfield region using dominant color but also segmented the playfield contour. So, these cues help to decide during and after the occlusion.

\section{THE PRoposed METHOD}

The proposed method tracked multiple objects in a scene using EKF and when they were occluded, color information was used to decide between objects. As the color information was integrated to Kalman filtering, the proposed method could efficiently track multiple objects under high occlusion. Fig. 1 shows the flowchart of the proposed method. The proposed method consists of four steps; background modeling, extended Kalman filtering, dominant color extraction and finally storing the tracked information. Comprehensive description of these steps follows.

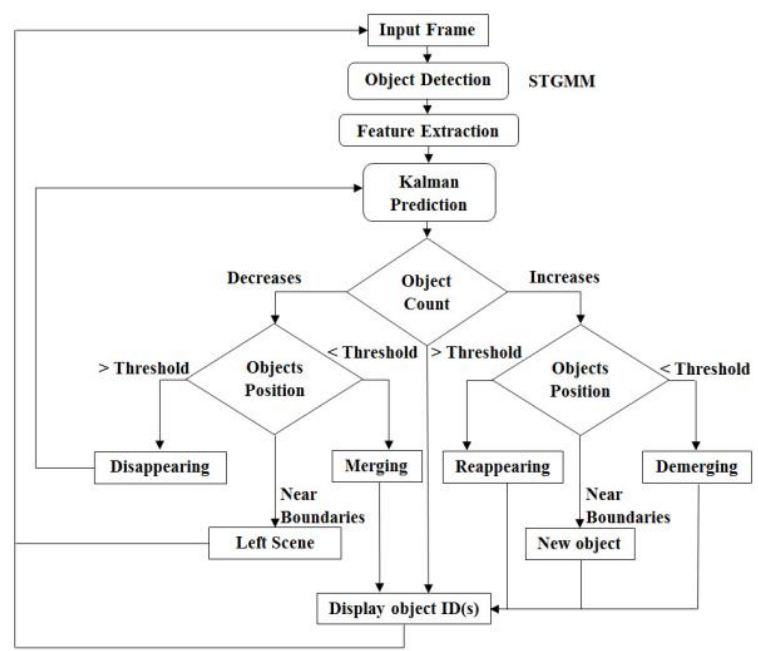

Fig. 1. The flowchart of proposed method.

\section{A. Background Modeling}

In this step, we review the STGMM proposed by Soh et al. [3]. The proposed method considers temporal behavior as well as spatial relations. Detailed explanation of the proposed
STGMM can be reviewed in [10].

\section{B. Extended Kalman Filtering with Past Information}

For tracking, we adopt EKF over linear Kalman filtering because most of the times the state variables and measurements are not linear combination of state variables, inputs to the system and noise. The key variables used in EKF were state estimate $\left(\hat{x}_{k}\right)$ and measurement $\left(z_{k}\right)$ whose relation can be depicted in Fig. 2. As, this is the advance research of our previous work so comprehensive explanation of EKF can be seen in [10].

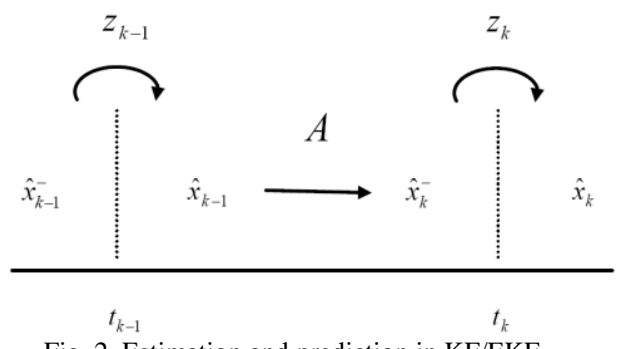

Fig. 2. Estimation and prediction in $\mathrm{KF} / \mathrm{EKF}$.

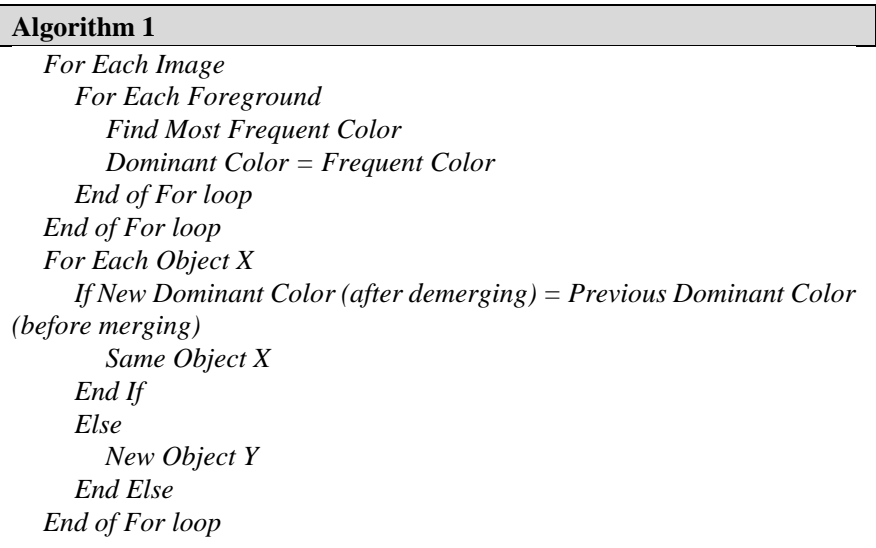

Algorithm 2 Merging \& Disappearing

For Each Object X

If ((Object Counter in Frame J-1 > Object Counter in Frame J) \&\& (No New Object Appears Near Boundaries))

If (Object Size in Frame J-Object Size in Frame J-1 > Threshold) End If

Store ID and Dominant Color in Merged Array

End If

Else

Blob Disappears

Store Center point, Dominant Color in Past Object Array End Else

End of For loop

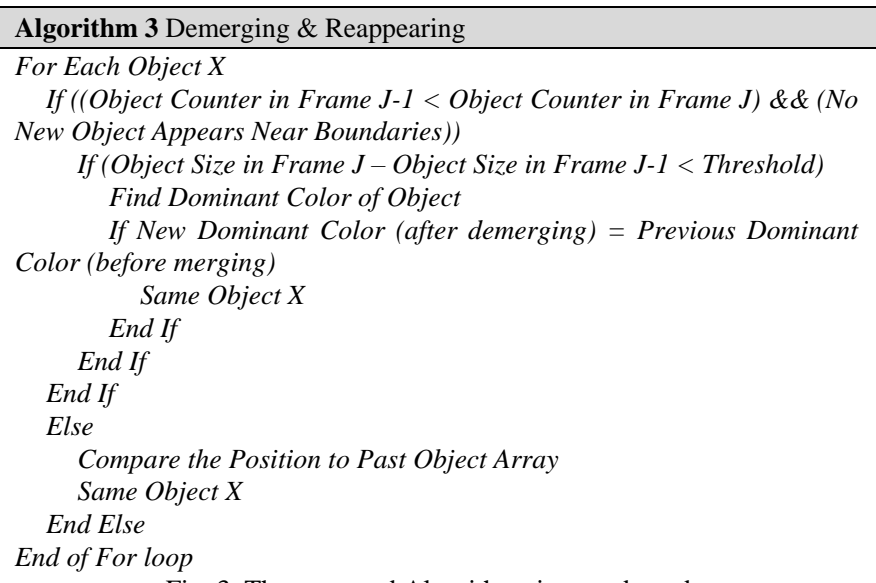

Fig. 3. The proposed Algorithms in pseudo code. 


\section{Extracting Dominant Color}

When objects were occluded and shown as merged blobs in STGMM, they were being treated as new objects by the conventional EKF. Therefore, we needed some invariant attributes i.e., object class and object color, to track objects under high occlusion. The foreground extracted from STGMM was taken into consideration. The RGB values of each pixel of the upper-half of the object were found and categorized into $n^{3}$ classes, where $\mathrm{n}$ equals 16 . For each object the histogram of most frequent color was found and then taken as invariant attribute of the object. Then using Bhattacharyya distance, the objects before merging were compared to the objects after the demerging to reassign their objects IDs.

The algorithms given in Fig. 3 clearly states the most occurring color of the object was extracted and used to compare at demerging to reassign tracking IDs correctly. The object disappeared and reappeared was also tracked with single unique ID throughout the scene.

\section{EXPERIMENTAL RESULTS}

\section{A. Extended Kalman Filtering with Past Information}

The experimental results are presented which shows the good tracking of moving independent and partial occluded objects. The direction of object was maintained to recover it's tracking ID after partial merging and past information for 10 frames to re-track object appearing after few frames by STGMM. The results are presented in Fig. 4 through Fig. 6. Left half represents proposed method and right is its STGMM.

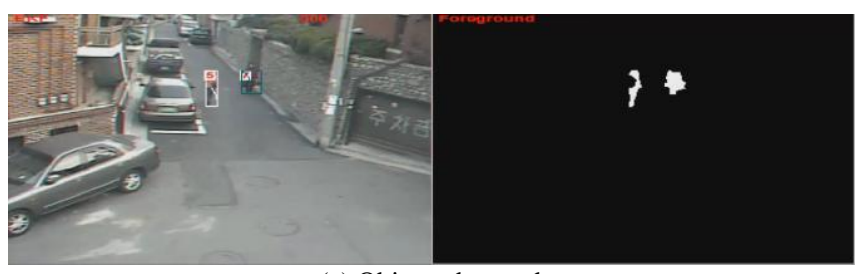

(a) Objects detected

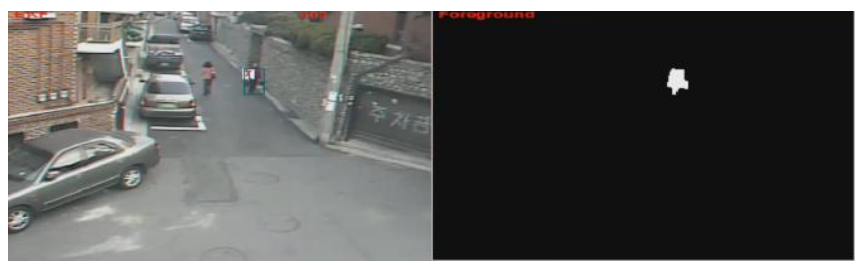

(b) Object disappearing in STGMM

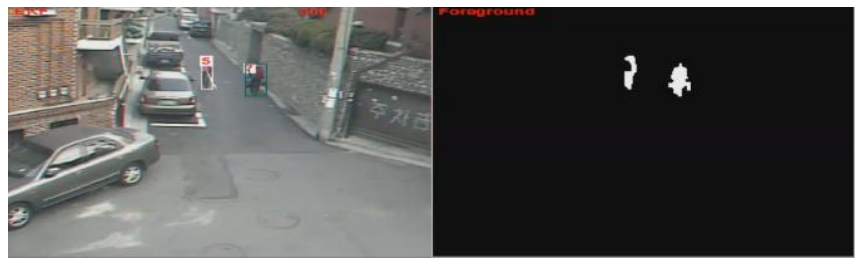

c) Object re-tracked with same tracking ID

Fig. 4. Experimental results of object disappearing, resolved with EKF using past information.

The Fig. 4 shows such a scenario where object with tracking ID 5 disappeared in (b), of $320 \times 240$ frame size video with frame rate 14 frames per sec, and reappeared in (c) in the STGMM. As, this object did not come from the boundaries of the frame so it should go with the same ID 5. It was resolved using past information of the objects.

The Fig. 5 shows partially occluding objects in (b) and separating in (c) retain tracking IDs. It is resolved using directions and past information of objects.

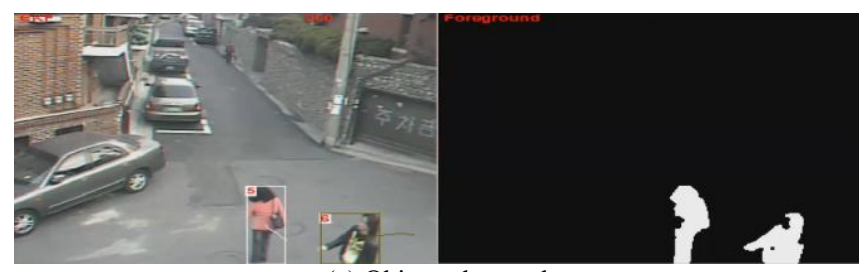

(a) Objects detected

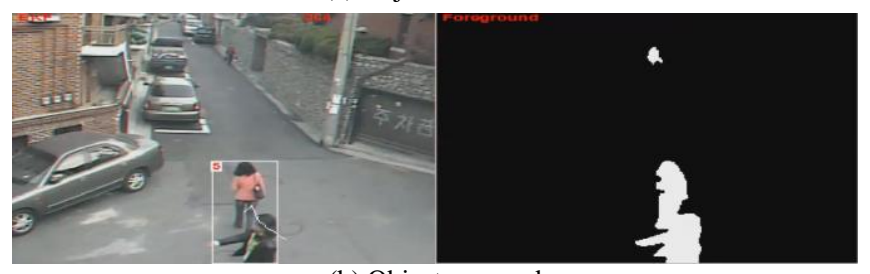

(b) Objects merged

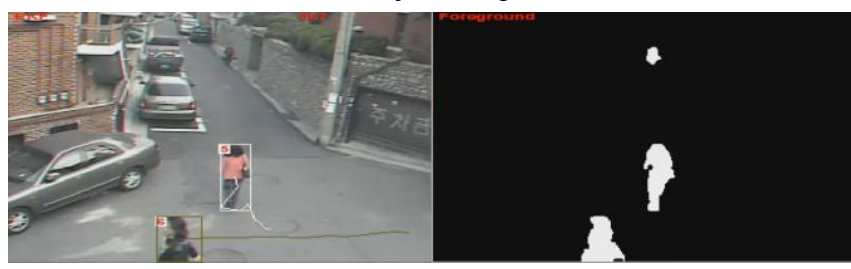

(c) Objects getting original tracking ID after demerging

Fig. 5. Experimental results of low occlusion, resolved with EKF using past information.

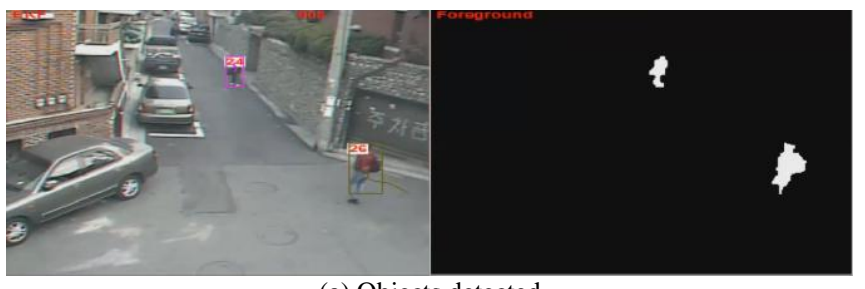

(a) Objects detected

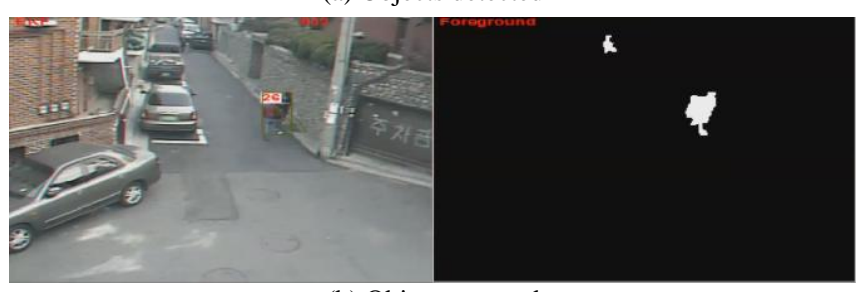

(b) Objects merged

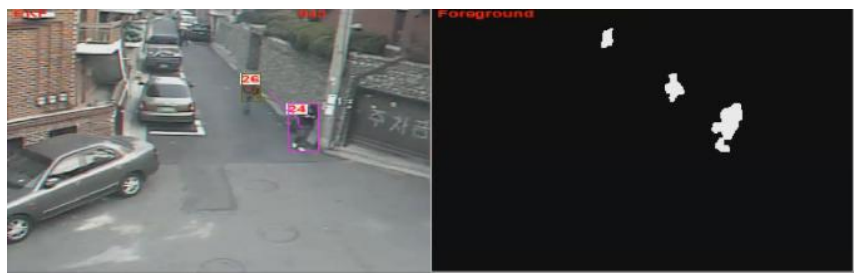

(c) Objects getting original tracking ID after demerging

Fig. 6. Experimental results of low occlusion, resolved with EKF using past information.

Fig. 6 shows the objects were highly occluded in (b) were represented as merged blobs in STGMM. As the objects were occluded for few frames and their directions were different so they were track-able using EKF without color otherwise it would not possible to track them with same tracking ID throughout the scene. Such problem is resolved in Fig. 8 and Fig. 9. 


\section{B. Extended Kalman Filtering with Past and Color Information}

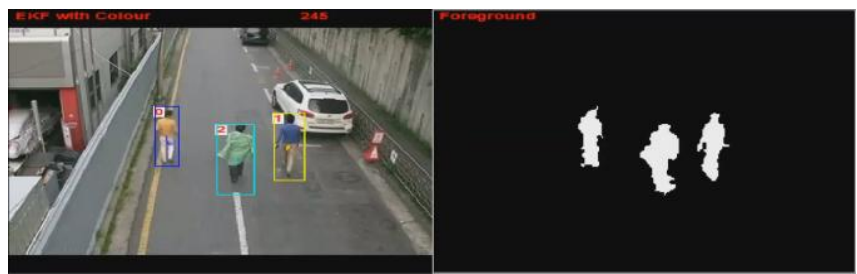

(a) Objects detected

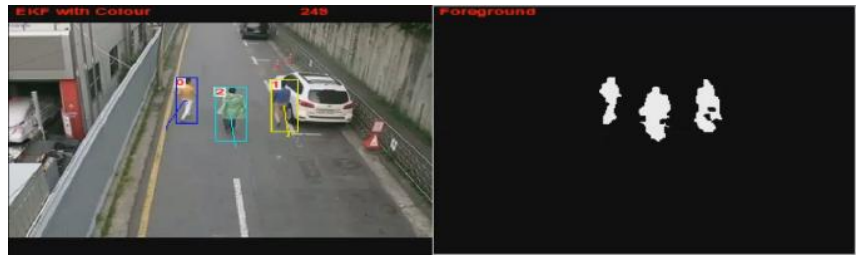

(b) Objects re-tracked after jump in video

Fig. 7. Experimental results of video error: frames missing, resolved with EKF using past information.

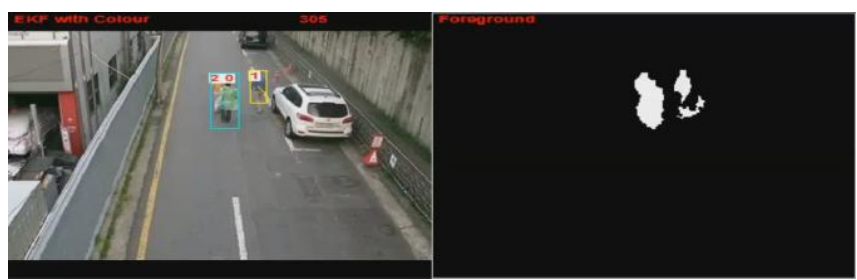

(a) Object broken in STGMM

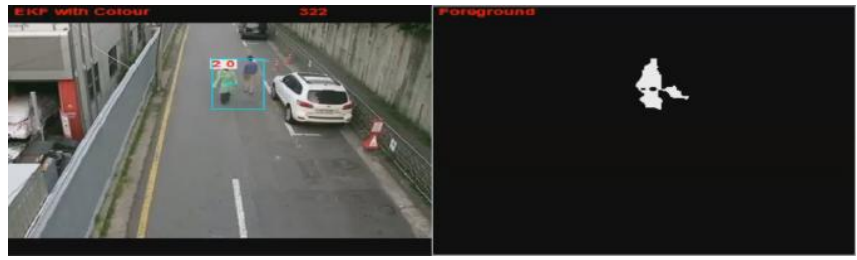

(b) Object disappeared in STGMM

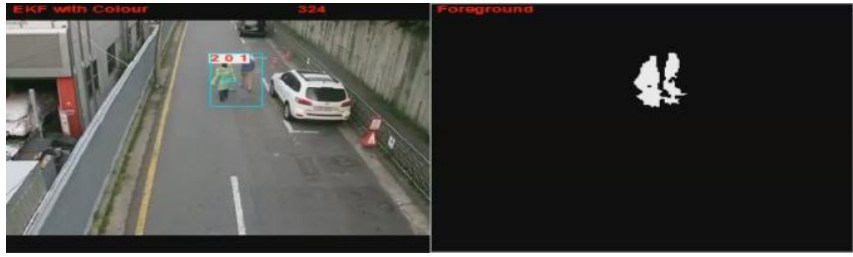

(c) Re-tracking all objects with original tracking IDs

Fig. 8. Experimental results of poor background modeling, resolved with EKF using past and color information.

Tracking highly occluded objects throughout the scene with same tracking ID requires invariant attributes such as object size and color. The Fig. 7 shows results for problem caused by camera error. The input video might be erroneous and camera might cause some errors in capturing videos for computer vision tracking algorithms. Such, problem is exploited in Fig. 7(a) and Fig. 7(b). The objects with tracking ID 0,1 and 2 in (a), of video $320 \times 240$ frame size with frame rate 30 frames per second, experienced a jump, as the objects in (b) appeared 55 pixels apart due to video capturing problem. They were re-tracked with same object IDs due to their invariant attributes i.e., size and color, including the direction and past information of the objects.

If the background modeling is compromised, the overall performance of tracking would be eventually compromised. The objects could disappeared in STGMM, and might break, reappear near or inside already occluded objects. To overcome such situations, invariant attributes provide good help in deciding between confusing objects. Fig. 8 deals and resolves such problems. In Fig. 8(a) the object with tracking ID 1 breaks in STGMM, the broken part should be regarded as noise or background, as presented in EKF with color result in Fig. 8(a). Fig. 8(b) shows the object with tracking ID 1 was occluded but disappeared in STGMM, and reappeared in STGMM as merged blob in Fig 8(c), so it is treated as same object as it was being tracked with ID 1 throughout the scene. Invariant attributes of the object helped to track with same tracking ID.

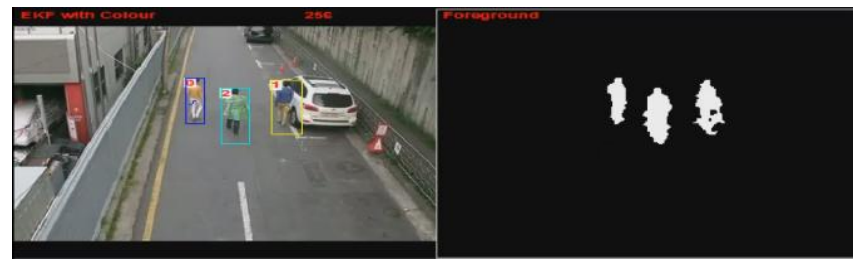

(a) Objects detected

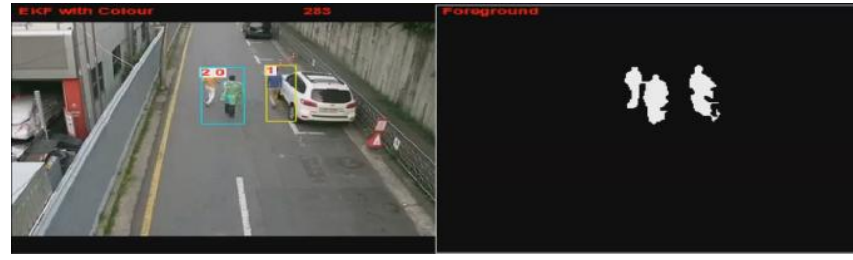

(b) Two of three objects merged

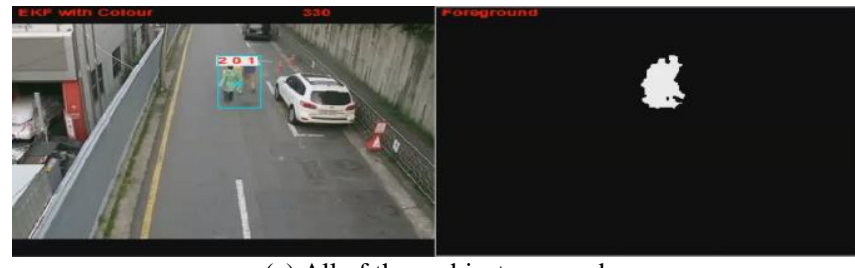

(c) All of three objects merged

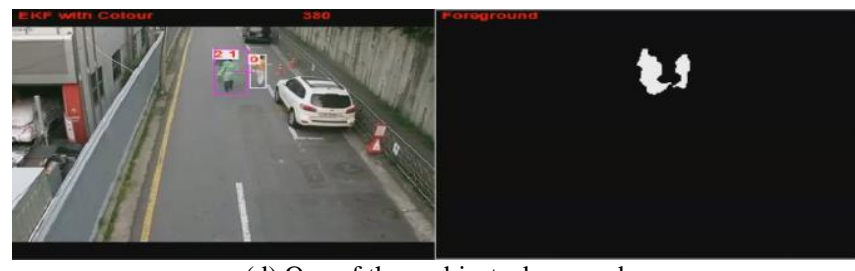

(d) One of three objects demerged

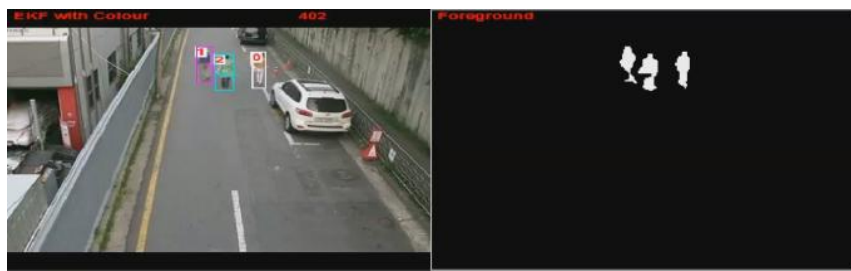

(e) All of three objects demerged

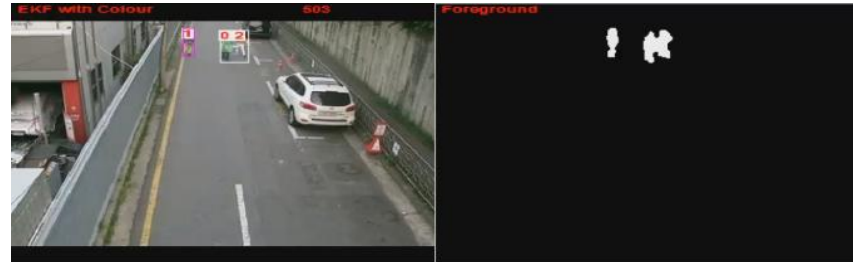

(f) Two of three objects re-merged

Fig. 9. Experimental results of high occlusion, resolved with EKF using past and color information.

The tracking of multiple objects occluded has been carried out in Fig. 9(a) through Fig. 9(f). The object with tracking ID 0,1 and 2 were independent in Fig. 9(a). Two i.e. 0 and 2, of three objects were occluded as shown in Fig. 9(b). 
The merging algorithm was called there and tracking IDs of occluded objects were shown in output. In Fig. 9(c) all the objects were occluded, so represented as merged object with all the tracking IDs on single merged object. The objects were close and overall size of object was increased with decrease in the object counter, so merging algorithm was satisfied. In Fig. 9(d) the object with tracking ID 0 demerged, so, by checking the dominant color of the demerged object, it got the object tracking ID 0 as before. Fig. 9(e) represents all independent demerged objects with correct object tracking ID and Fig. 9(f) shows objects with tracking ID 0 and 2 re-merges.

\section{CONCLUSION}

Multi-target tracking was done using EKF with past information of objects when there were partially occluded and also disappeared and reappeared by poor background modeling. When the objects were highly occluded, invariant attributes like color and size were integrated to EKF with past information to resolve tracking challenges.

In future, we will also be investigating the behavior and event of the object associated to tracking. Segmentation of horizontal merged objects in STGMM would be taken into consideration. Also, we would like to track multiple objects across the viewpoints using single dome camera based on Kalman prediction, past and color information.

\section{REFERENCES}

[1] J. L. Xing, H. Z. Ai, L. W. Liu, and S. H. Lao, "Multiple player tracking in sports video: A dual-mode two-way bayesian inference approach with progressive observation modeling," IEEE Transaction on Image Processing, pp. 1652-1667, June 2011.

[2] Y. Benezeth, P. Jodoin, B. Emile, H. Laurent, and C. Rosenberger, "Review and evaluation of commonly-implemented background subtraction algorithms," in Proc. 19th International Conference on Pattern Recognition, 2008, pp. 1-4.

[3] Y. S. Soh, Y. S. Hae, and I. Kim, "Spatio-temporal gaussian mixture model for background modeling," in Proc. 2012 IEEE International Symposium on Multimedia (ISM), Dec. 2012, pp. 360-363.

[4] S. J. Julier and J. K. Uhlmann, "Unscented filtering and nonlinear estimation," in Proc. the IEEE, 2004, pp. 401-422.

[5] C. Y. Liu, P. L. Shui, and S. Li, "Unscented extended Kalman filter for target tracking," Journal of Systems Engineering and Electronics, pp. 188-192, April 2011

[6] J. Berclaz, F. Fleuret, E. Turetken, and P. Fua, "Multiple object tracking using K-Shortest paths optimization," IEEE Transactions on Pattern Analysis and Machine Intelligence, pp. 1806-1819, 2011.

[7] Y. Zhai, M. B. Yeary, S. Cheng, and N. Kehtarnavaz, "An object-tracking algorithm based on multiple-model particle filtering with state partitioning," IEEE Transactions on Instrumentation and Measurement, pp. 1797-1809, 2009.

[8] Z. H. Khan, I. Y.-H. Gu, and A. G. Backhouse, "Robust visual object tracking using multi-mode anisotropic mean shift and particle filters," IEEE Transactions on Circuits and Systems for Video Technology, pp. 74-87, 2011.
[9] L. Y. Li, W. M. Huang, I. Y.-H. Gu, R. J. Luo, and Q. Tian, "An efficient sequential approach to tracking multiple objects through crowds for real-time intelligent CCTV systems," IEEE Transactions on Systems, Man, and Cybernetics, Part B: Cybernetics, pp. 1254-1269, 2008.

[10] I. Kim, M. M. Khan, T. W. Awan, and Y. S. Soh, Multi-Target Tracking Using Color Information.

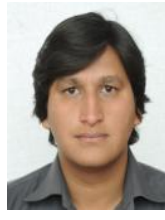

Malik Muhammad Khan was born in Lahore, Pakistan on Nov. 8, 1988. He got BS in electrical engineering in 2011 from Govt. College University in Lahore, Pakistan. He entered a master course in information and communication engineering in Myongji University in 2012.

His current interest of research includes vehicle to grid, Kalman filtering, background modeling and multi-viewpoint tracking.

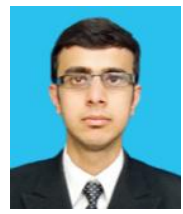

Tayyab Wahab Awan was born in Peshawar, Pakistan on Aug. 17, 1990. He got BS in telecommunication engineering in 2012 from National University of Computer and Emerging Sciences in Peshawar, Pakistan. $\mathrm{He}$ entered a master course in information and communication engineering in Myongji University in 2013.

His current interest of research includes vehicle to grid, Kalman filtering, particle filtering and multi-viewpoint tracking.

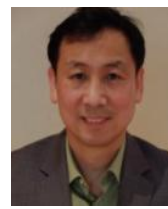

Intaek Kim was born in Seoul, Korea in 1960. He received BS and MS in electronics engineering from Seoul National University in Seoul, Korea in 1980 and 1984 respectively. He obtained $\mathrm{PhD}$ in electrical engineering from Georgia Institute of Technology in Atlanta, Georgia, USA in 1992

He worked for Goldstar central research lab from 1993 to 1995 as a senior engineer and joined Myongji University from 1995. He is now a professor in the Dept. of Information and Communication Engineering. His recent publications deal with the area of face recognition, hypersepctral image and MR imaging.

His research interest includes pattern recognition, image processing and smart grid area.

Prof. Kim is a member of Korean Institute of Electronics Engineer.

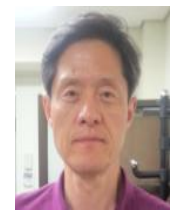

Youngsung Soh was born in Seoul, Korea on Mar. 4 1956. He got BS in electrical engineering in 1978 from Seoul National University in Seoul, Korea. He obtained MS and $\mathrm{PhD}$ in computer science from the University of South Carolina in Columbia, South Carolina, USA in 1986 and 1989 , respectively.

He served in the Korean army from June 1980 to Sept. 1982. He worked in Systems Engineering Research Institute in Korea as a senior researcher from Sept. 1989 to Feb. 1991. He joined Myongji University in Korea from Mar. 1991 and is currently a full professor in the Dept. of Information and Communication Engineering.

His current interest of research includes object tracking, stereo vision, and parallel algorithms for image processing.

Prof. Soh is a member of Korea Information Processing Society and Korea Signal Processing Systems Society. 